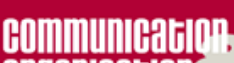

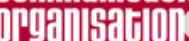

Communication et organisation

Revue scientifique francophone en Communication organisationnelle

56 | 2019

Les organisations malades du numérique

\title{
HINAULT Anne-Claude, OSTY Florence et SERVEL Laurence, 2019. Enquêter dans les organisations. Comprendre pour agir
}

Rennes : Presses Universitaires de Rennes. Coll. « Didact sociologie »

\section{Ugo Roux}

\section{OpenEdition}

\section{Journals}

Édition électronique

URL : https://journals.openedition.org/communicationorganisation/8547

DOI : 10.4000/communicationorganisation.8547

ISSN : 1775-3546

\section{Éditeur}

Presses universitaires de Bordeaux

Édition imprimée

Date de publication : 1 décembre 2019

Pagination : 157-158

ISBN : 979-10-300-0571-4

ISSN : 1168-5549

Référence électronique

Ugo Roux, «HINAULT Anne-Claude, OSTY Florence et SERVEL Laurence, 2019. Enquêter dans les organisations. Comprendre pour agir », Communication et organisation [En ligne], 56 | 2019, mis en ligne le 01 décembre 2019, consulté le 24 mars 2023. URL : http://journals.openedition.org/ communicationorganisation/8547 ; DOI : https://doi.org/10.4000/communicationorganisation.8547 


\section{Recensions}

HINAULT, Anne-Claude, OSTY, Florence et SERVEL, Laurence, 2019. Enquêter dans les organisations. Comprendre pour agir. Rennes: Presses Universitaires de Rennes. Didact sociologie. ISBN 978-2-7535-7687-2

Anne-Claude Hinault, Florence Osty et Laurence Servel nous proposent un ouvrage intéressant, en ce sens qu'il se distingue du vaste corpus traitant des diverses méthodologies employées en sciences humaines et sociales. En effet, si ce dernier est essentiellement composé de livres orientés vers une finalité de recherche et de production scientifiques, le manuel ici recensé propose une démarche et des repères inédits pour mener des enquêtes sociologiques lors de situations d'intervention et d'action dans des organisations. Comme indiqué sur la quatrième de couverture : "Au-delà des repères méthodologiques constitutifs d'une démarche qualitative de recherche, ce manuel envisage l'enquête comme une expérience à vivre. Il invite à développer une posture réflexive d'analyse de la pratique et de la relation au terrain. »

L'ouvrage se divise en sept chapitres qui reprennent les étapes majeures d'une démarche d'enquête qualitative. La première partie - "Résister à la commande, coconstruire la demande »- «traite des enjeux de la relation au commanditaire et de l'analyse de la demande " (p. 14-15). La deuxième partie, - "Préparer son enquête, se préparer à faire enquête »- donne des clés pour préparer une enquête de terrain, et ce « en mettant l'accent sur l'enjeu et les méthodes d'exploration comme sur l'exploitation des matériaux de pré-enquête pour parvenir à problématiser la recherche. » (p. 51-52). Le deuxième enjeu de ce chapitre est d'aider le lecteur à se projeter dans le rôle d'enquêteur, à s'interroger sur sa posture et à maintenir un équilibre entre engagement et distanciation.

La troisième partie - «Conduire les entretiens, se mettre à l'écoute » - propose de se pencher sur le déroulement d'une enquête par entretiens en se focalisant sur quatre points pour traiter au mieux la problématique et les hypothèses ; à savoir, l'organisation de l'enquête, la construction des entretiens, leur conduite et leur transformation en matériaux pertinents pour une analyse. La quatrième partie - «Conjuguer les méthodes, surmonter les épreuves " - s'attache à présenter des méthodes complémentaires à l'entretien pour recueillir des matériaux à même d'enrichir l'enquête. De plus, ce chapitre propose également d'examiner «les formes d'engagement du commanditaire mais aussi celles des interviewés et du sociologue.»(p. 112).

La cinquième partie - «Analyser les données, tisser les fils de l'interprétation »fournit des points d'appui méthodologiques pour appréhender et traiter les nombreuses données produites lors de l'enquête. La sixième partie - « Restituer l'enquête : tendre 
un miroir, soutenir l'approbation » - explore quatre étapes majeures de la restitution de l'enquête ; à savoir, la mise en forme de l'analyse sociologique, l'anticipation des dynamiques d'écoute et de réception de l'analyse, l'élaboration d'une stratégie de restitution et le recueil de nouvelles données et pistes d'interprétation. La septième partie - "Identifier un devenir possible, soutenir un changement souhaitable »développe une méthode pour problématiser le changement, c'est-à-dire dégager des données récoltées ce qui représente un enjeu en termes de changement, défendre une stratégie d'intervention et la déployer.

Finalement, Anne-Claude Hinault, Florence Osty et Laurence Servel signent un ouvrage qui s'adresse aussi bien aux étudiants et chercheurs en SHS qu'aux praticiens qui ont recours à l'enquête dans leurs missions. Les auteures reprennent, à travers une écriture agréable, des expériences parlantes et des repères pratiques, des cadres théoriques et des méthodologies essentiellement éprouvées dans le domaine de la recherche fondamentale pour leur donner une dimension appliquée et utilitaire au sein des organisations.

Pour conclure sur une note pratique, le lecteur appréciera la présence de fiches-outils qui ponctuent parfois le texte et qui contiennent de nombreux conseils pour préparer ses enquêtes ainsi que la présence des multiples exemples empiriques, issus de terrains variés, qui illustrent judicieusement çà et là les propos des auteures. Dans la même veine, le lecteur remarquera, au-delà de la classique table des matières, une table de ces fiches-outils et de ces exemples.

Ugo ROUX

Ugo Roux est docteur en Sciences de l'Information et de la Communication. Il est actuellement attaché temporaire d'enseignement et de recherche au sein du département information-communication option métiers du livre et du patrimoine de l'IUT d'Aix-Marseille Université. Il est également membre de l'Institut Méditerranéen des Sciences de l'Information et de la Communication (IMSIC) - EA 7492. Courriel : ugo.roux@univ-amu.fr 